UNITED STATES DEPARTMENT OF THE INTERIOR GEOLOGICAL SURVEY

\title{
A Hewlett-Packard 9830A BASIC language program for Plumbotectonics
}

by

\author{
Robert E. Zartman and Bruce R. Doe \\ U.S. Geological Survey \\ Denver, Colorado 80225
}
Open-File Report 80-1088
1980

\author{
Use of trade names is for descriptive \\ purposes only and does not constitute \\ endorsement by the U. S. Geological Survey
}


Three broad geologic environments, or reservolrs, having appreciable amounts of $\mathrm{U}$, Th, and $\mathrm{Pb}$ and sufficlent longevity, are required by the plumbotectonics model. These resevoirs, the mantle, upper continental crust, and lower continental crust, and an additional short-lived mixing orogene provide the necessary chemical environments to produce gross terrestrial 1sotopic patterns. The transfer of matter among reservolrs is treated as proceeding through cycles--called orogenies--of discrete, sequential operations that can be expressed by a series of BASIC program statements (paralleling the algebraic equations of the companion paper). From the sets of numerical data generated as matrix arrays, both the total mass distribution and the $\mathrm{U}$, $\mathrm{Th}$, and $\mathrm{Pb}$ 1sotopic contents among reservolrs can be determined as a function of time.

Two versions of the model are presented by Zartman and Doe (in press), which follow the same basic program form and differ only in certain parametric values. We 11st version $I$ in 1 ts entirety in Appendix $A$, and supply the pertinent substitute lines for conversion to version II in Appendix $B$. In addition to yielding mass distribution and $\mathrm{U}$, Th, and $\mathrm{Pb}$ isotopic contents of reservoirs, several output options are available for calculating isotopic ratios, mean age of the crust, and isotope concentrations of reservoirs. A separate subroutine is also provided, which modifies the main program, for computing the mean age of isotopes in the crust.

The program was written specifically for use with the Hewlett-Packard model $9830 \mathrm{~A}$ minicomputer with $8 \mathrm{~K}$ words of memory, but with a few modifications it could be adapted to several other comparable H-P models. If the appropriate tape cartridge is sent to us at Ma1lstop 963, Federal enter, Denver, Colorado, 80225, we can supply you with a copy of the program. 


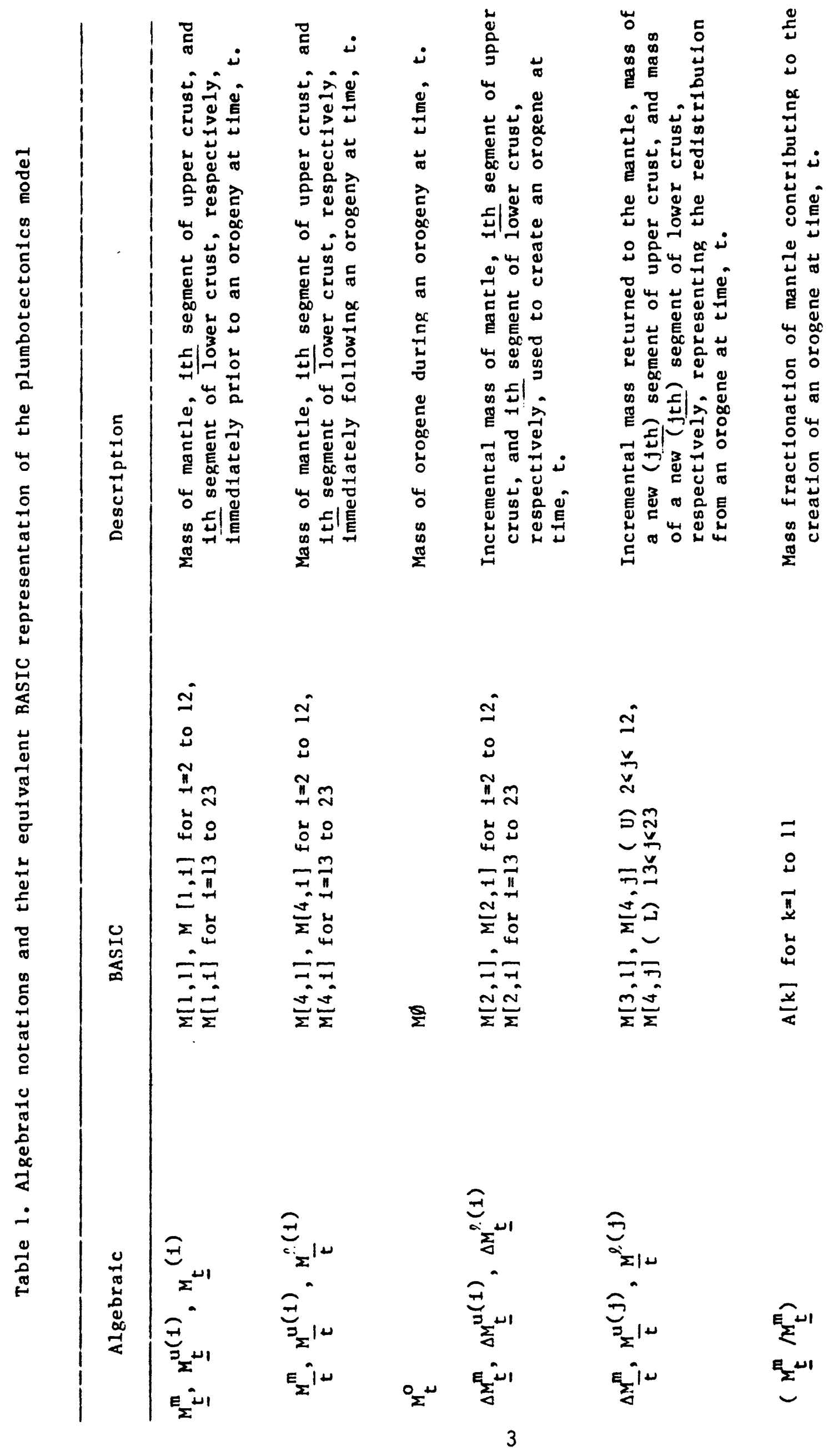




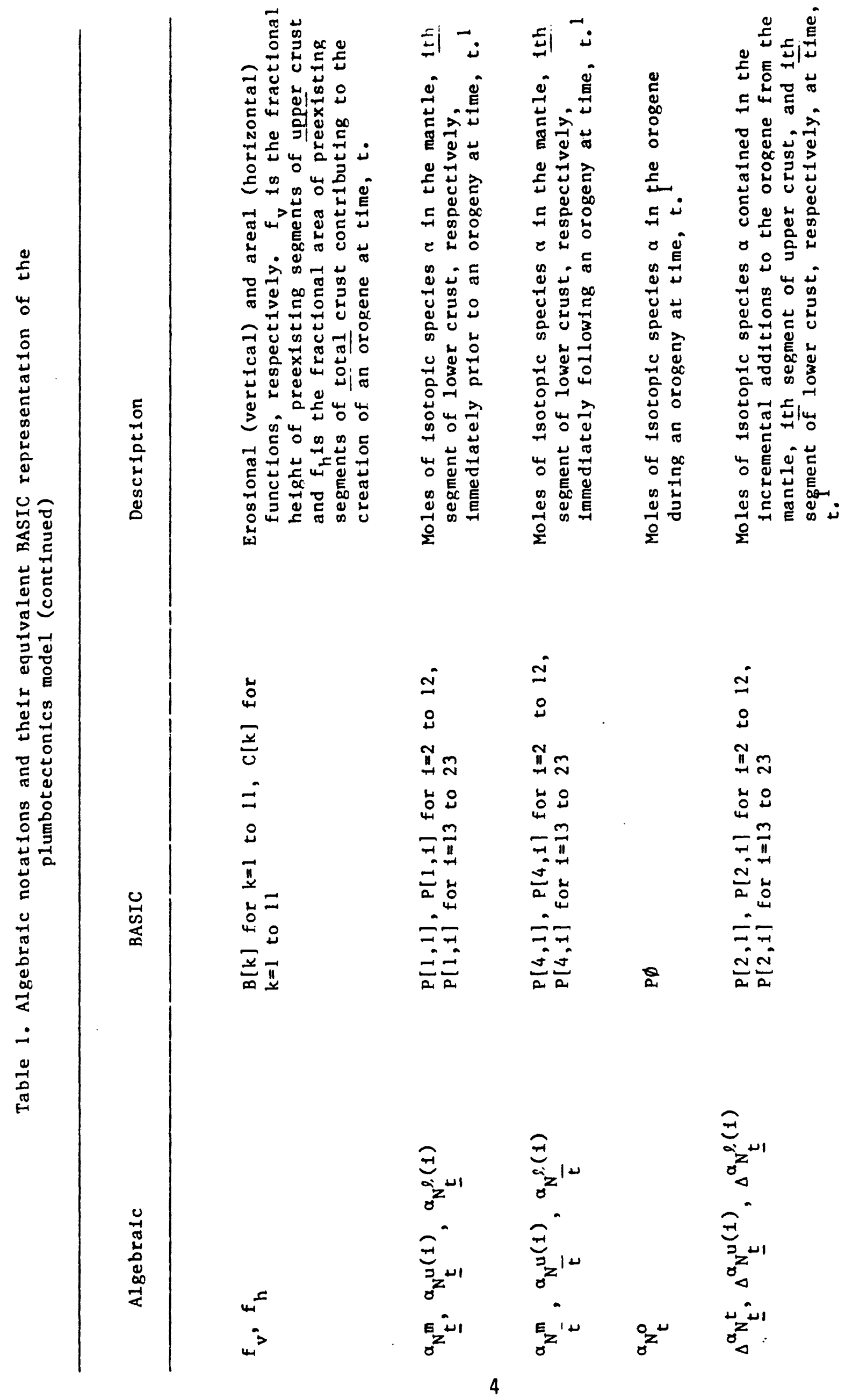




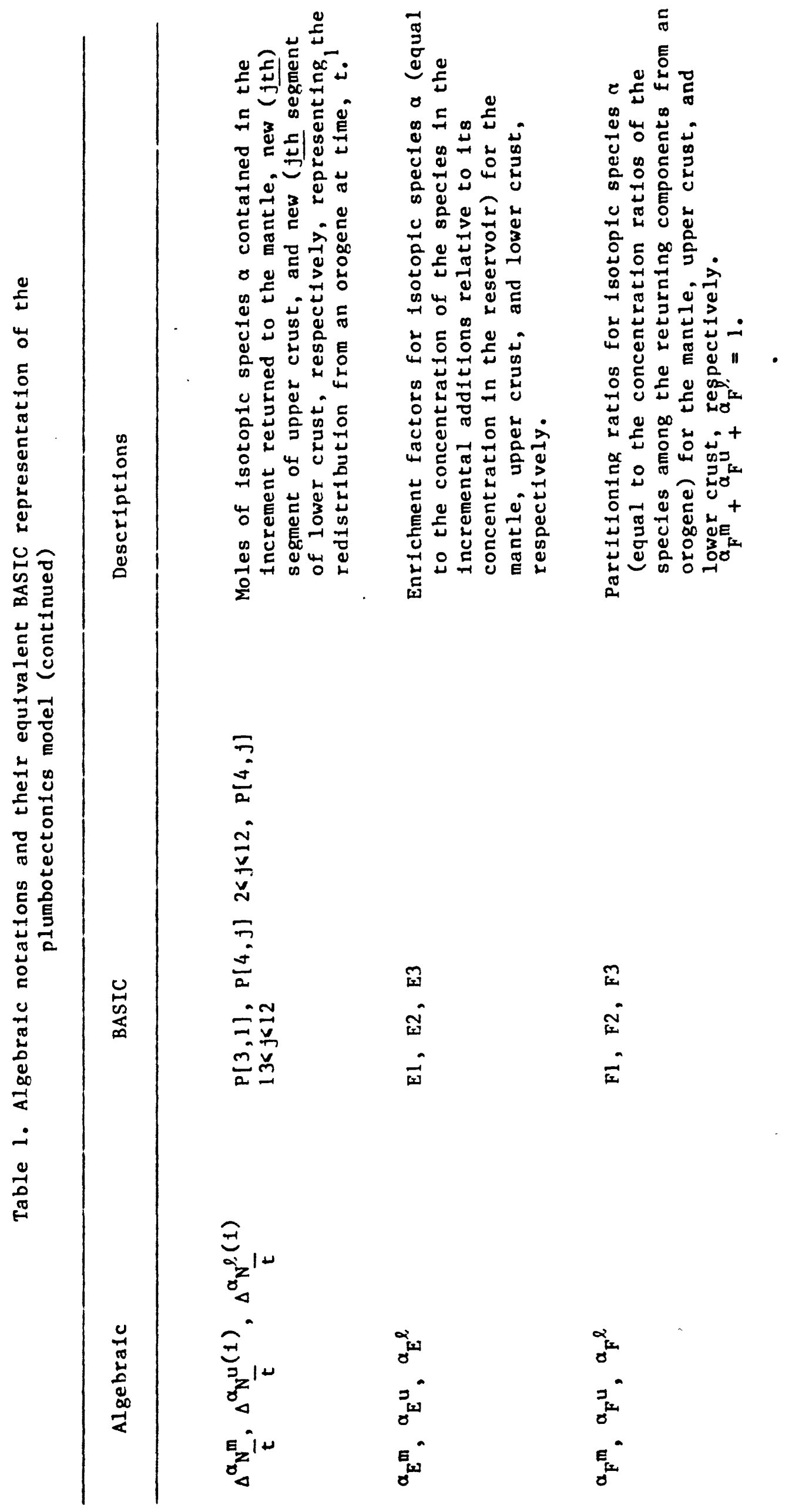




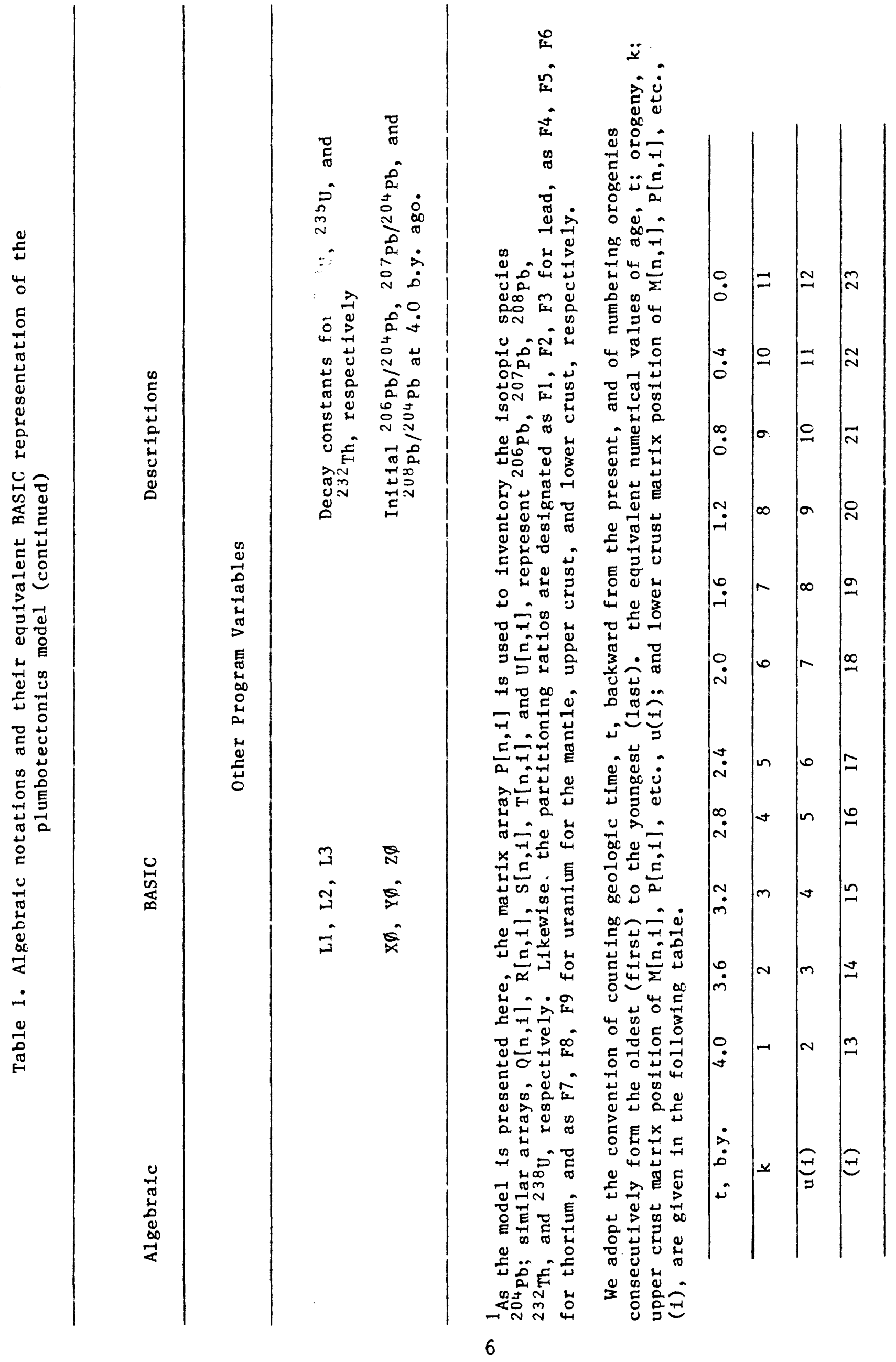




\section{PROGRAM EXPLANATION}

Each version ( $I$ and II) of the main plumbotectonics program, including its five output options (1. = Reservoir mass and isotope content, $2 .=$ Isotopic ratios, 3. $=\mathrm{T}, \mathrm{Max}, 4 .=\mathrm{T}, \mathrm{Min}$, and $5 .=$ Reservoir concentrations), is stored as a self-contained file on a tape cartridge, which will operate Independently of any auxiliary files. In this form one needs only to activate the program by issuing the RUN command, and then choosing the desired output option. The corresponding version I and II subroutine programs for calculating mean age of isotopes in the crust require substantial modification of the main program, and are themselves also given the status of selfcontained files. more general form of the program can easily be created whereby frequently changed numerical values of certain model parameters are stored as separate data files or supplied as input when the program is run.

A brief synopsis of the main program designating lines and functions is provided by the following table.

\section{LINES}

$10-70,290-400$, $550-780$

$80-280,410-540$

$790-2020$

$2030-2190$

$2200-2590$

$2600-3250$

\section{FUNCTION}

Initlalize conditions and dimension arrays.

Numerical evaluation of model parameters.

Functions for calculating mass and isotope contents of reservoirs.

Output options and directory.

Subroutine for reservoir mass and isotope content.

Subroutine for isotopic ratios. 

T,Max.

$3430-3770$

Subroutine for minimum mean age of crust, T,Min.

$3780-4040$

Subroutine for 1sotope concentrations of reservoirs.

An outline of the plumbotectonics model with the various parameters assigned their appropriate numerical values is present for version I in Table 2 and for version II in Table 3. From this description, all the BASIC variables for each version is listed in Table 4 can be evaluated, and the program can be run straightforwardly with the only remaining decision being that of the output option desired. Some selected results directly obtained from the model (or slight modifications thereof, such as the calculation of average element concentrations in parts per million are given in appendices $A$ and $B$ following the program listings. The reader is referred to the companion paper of Zartman and Doe (in press) for a discussion of the Implications of these results to terrestrial lead isotopic patterns.

\section{REFERENCES}

Doe, B. R., and Zartman, R. E., 1979, Chapter 2. Plumbotectonics I, The Phanerozoic, in Geochemistry of Hydrothermal Ore Deposits, 2nd Edition (H. L. Barnes, ed.), New York, Wiley-Interscience, p. 22-70. Zartman, R. E., and Doe, R. R., in press, Plumbotectonics--The model: Tectonophysics. 


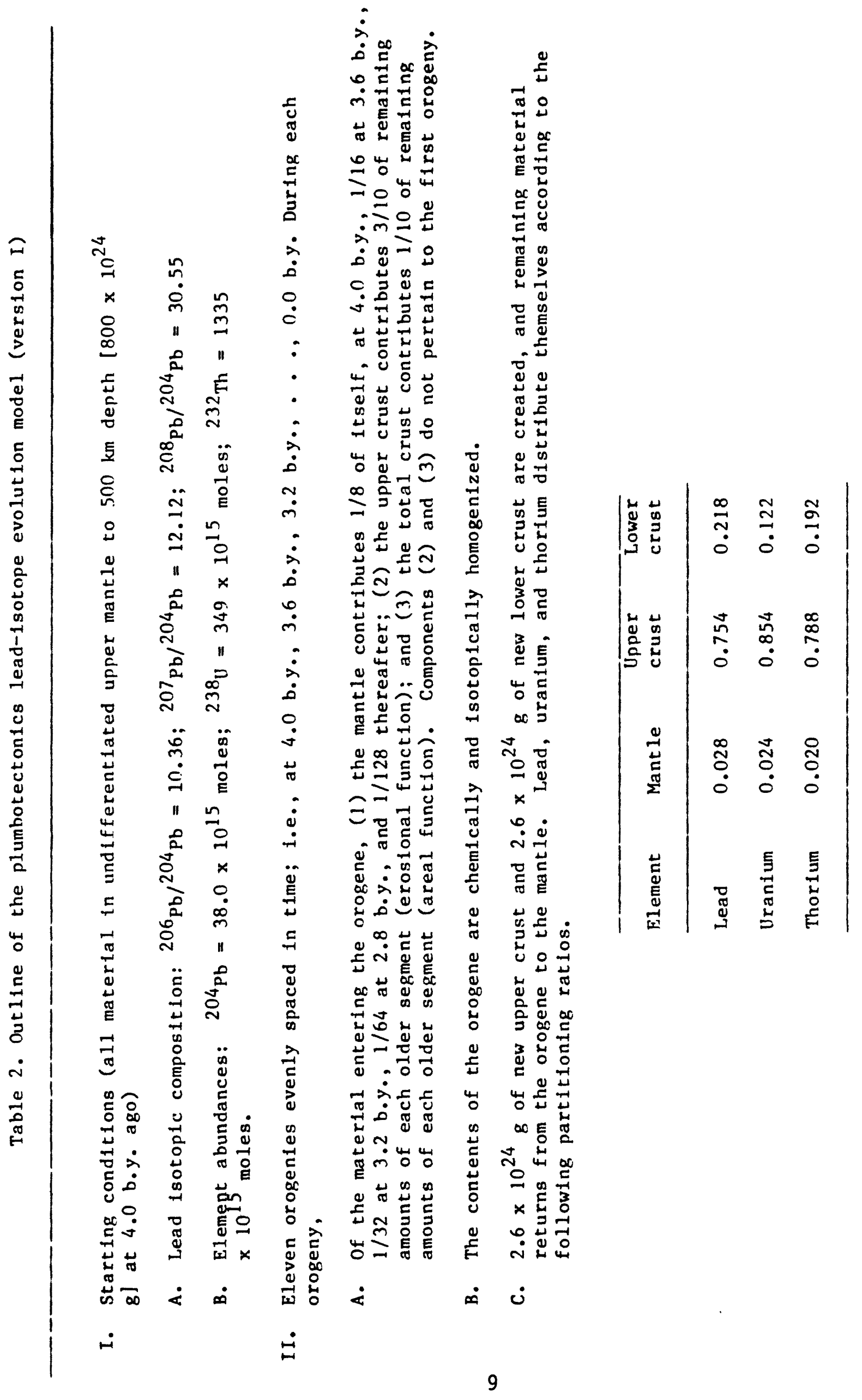




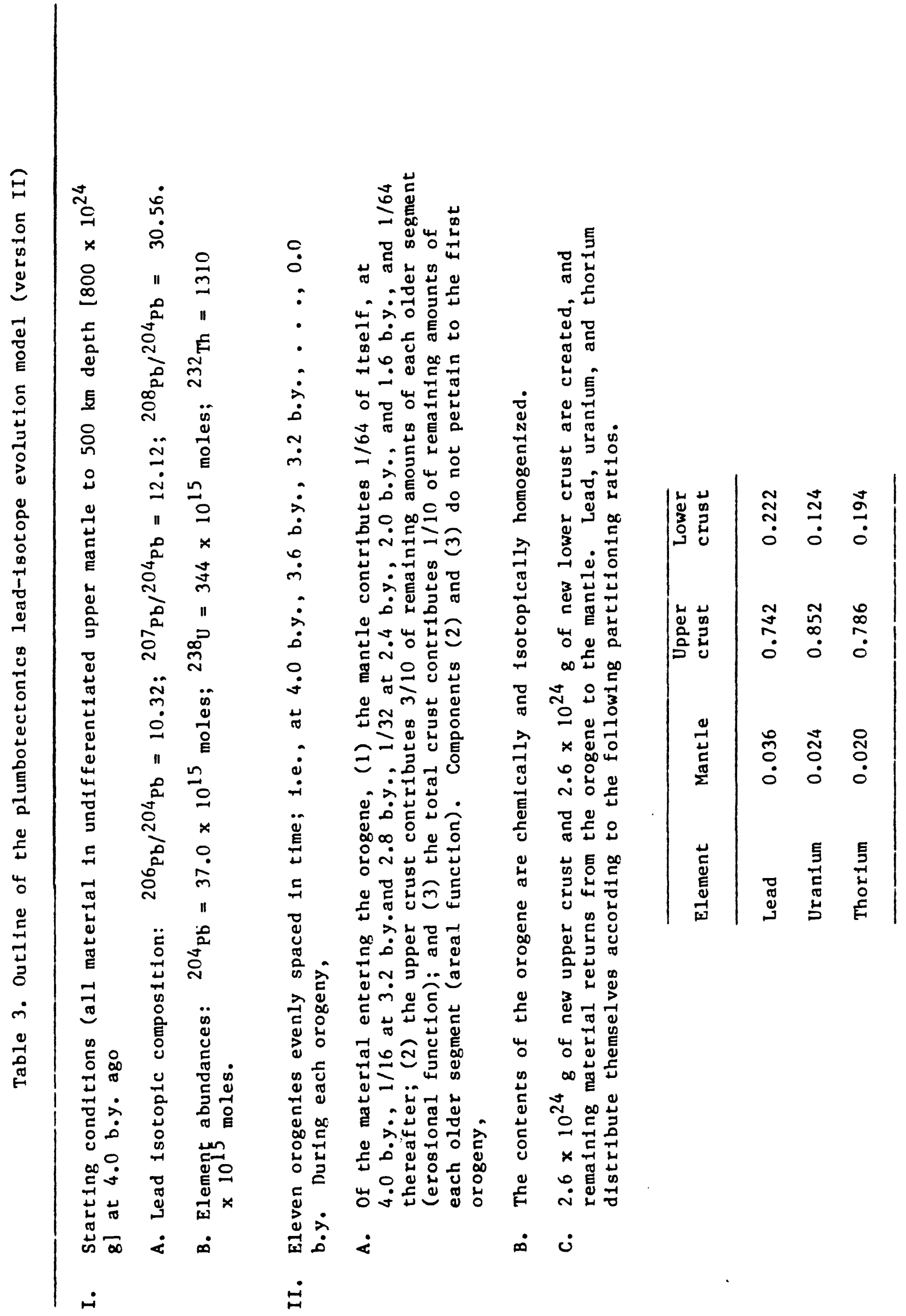




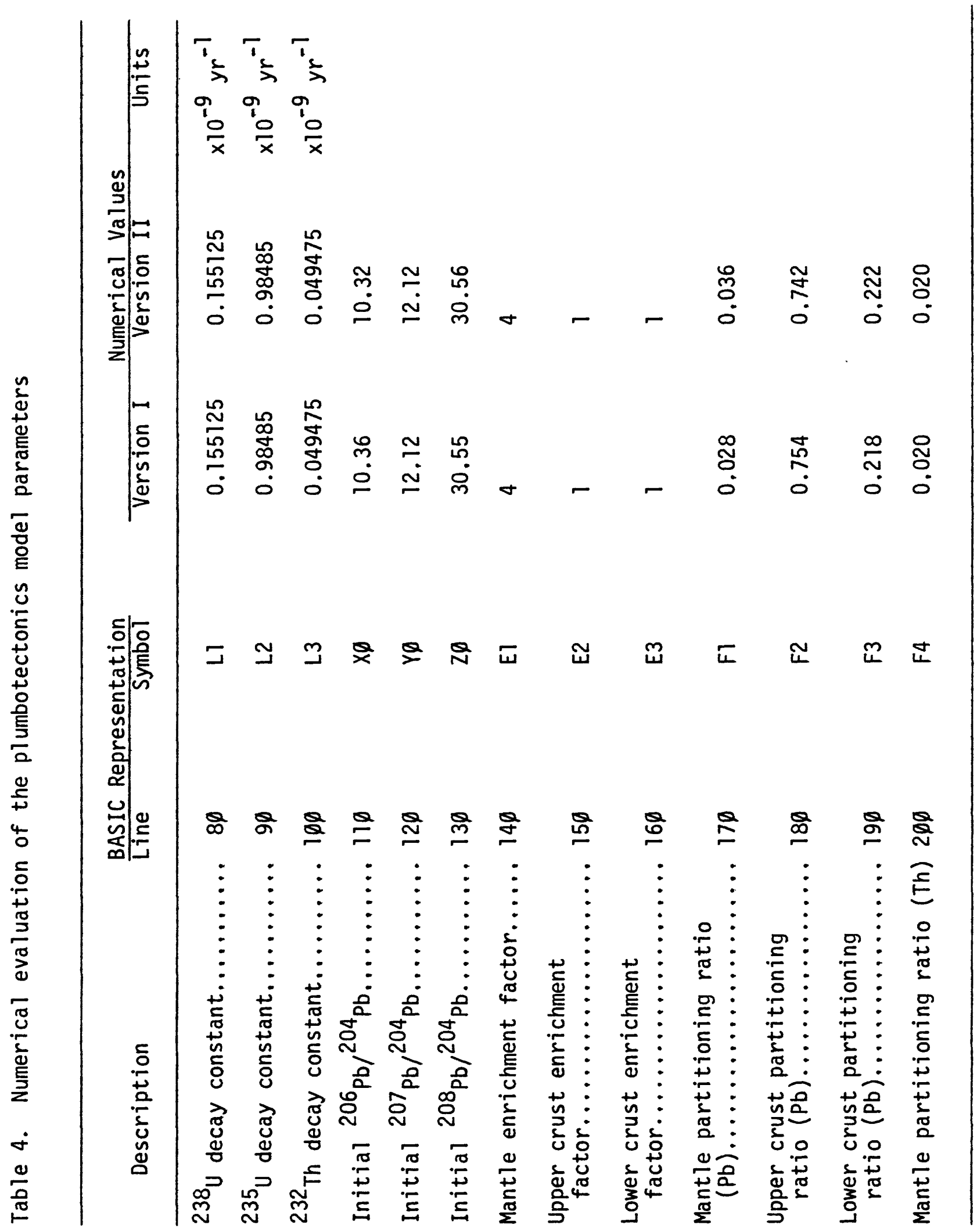




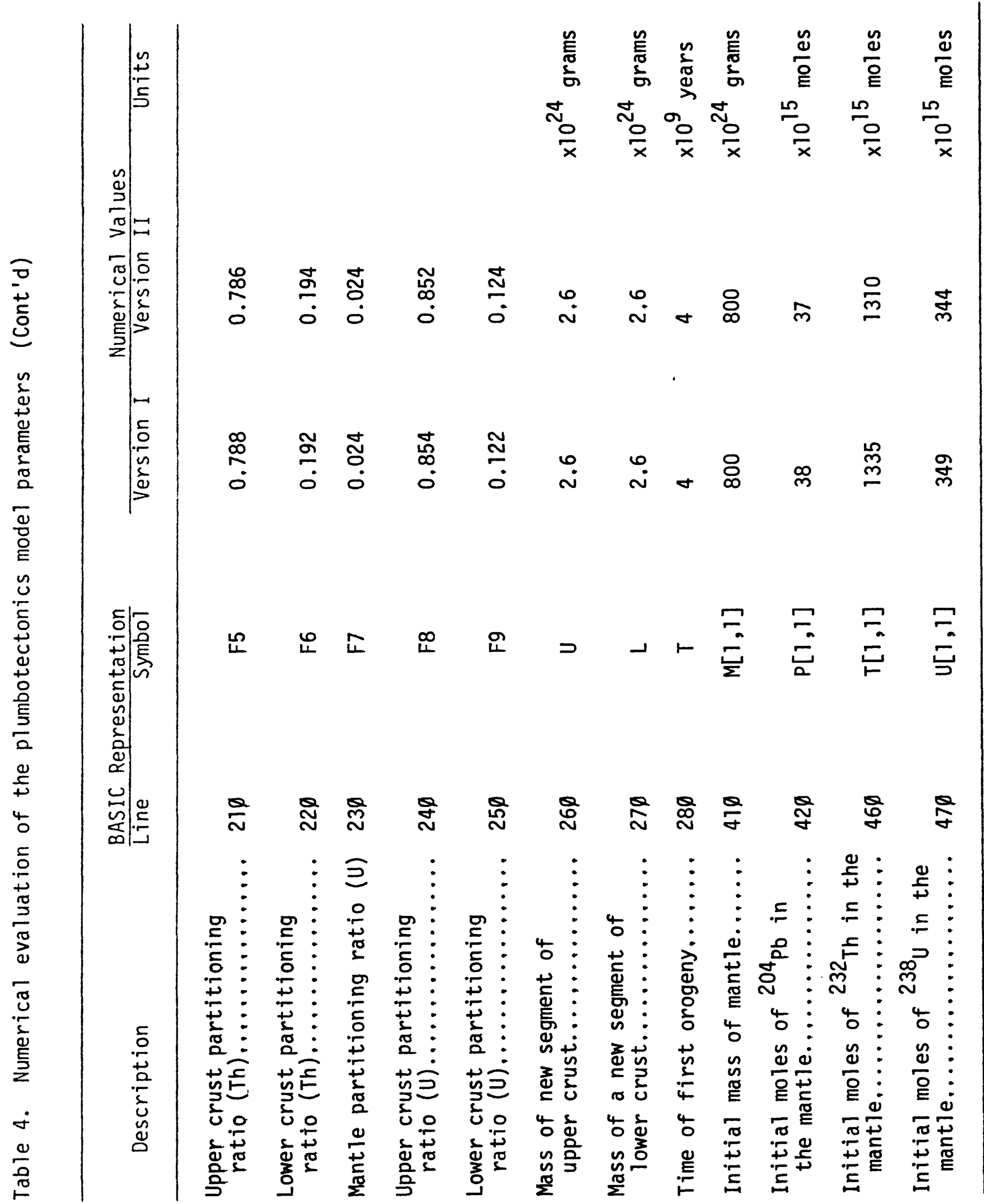




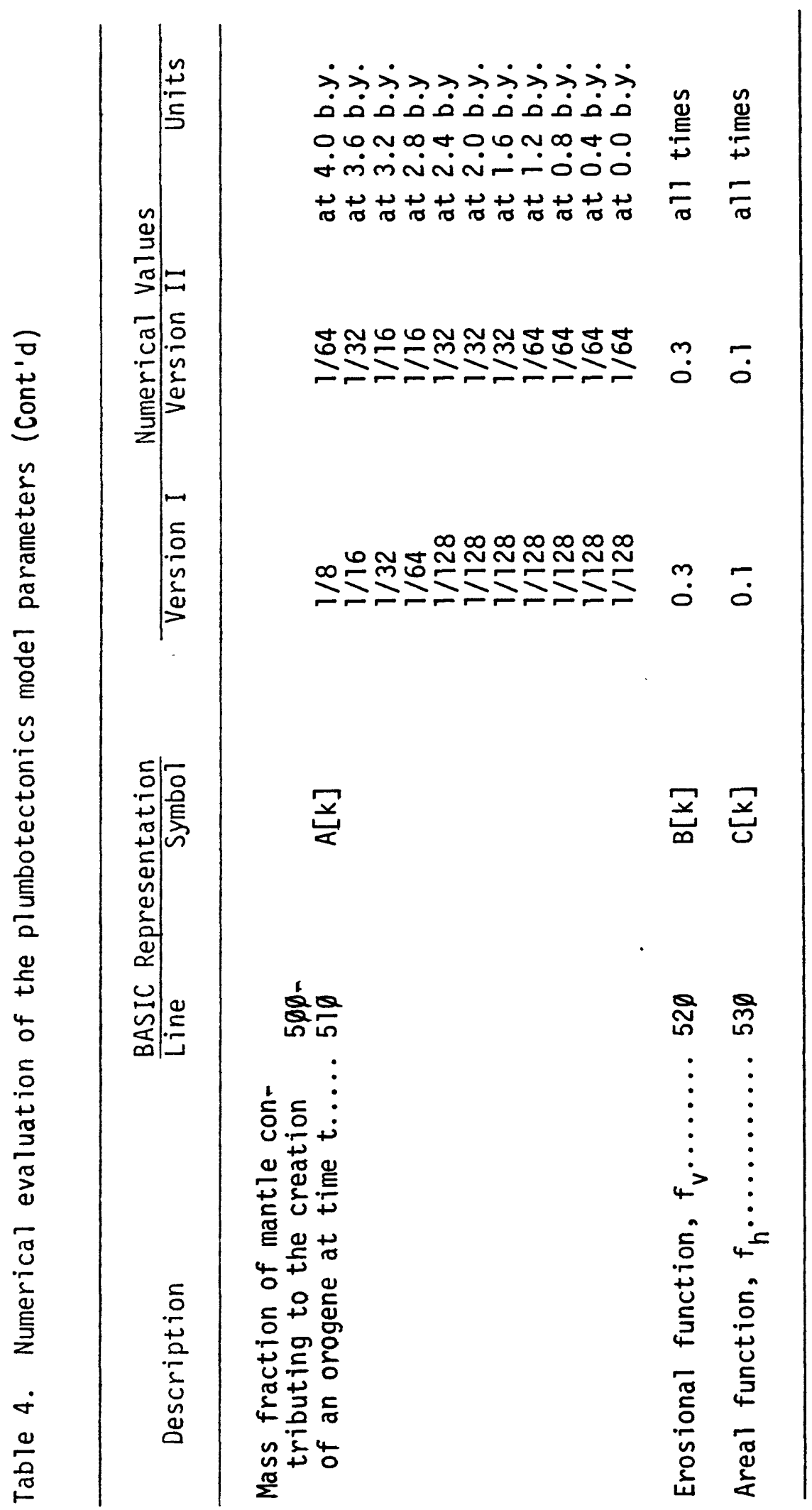


FFFEHIT: A: FLUHEOTECTOHICS MUIEL CWEFSIOH I)

1. LIETIHE DF MAIA FEOIFAM

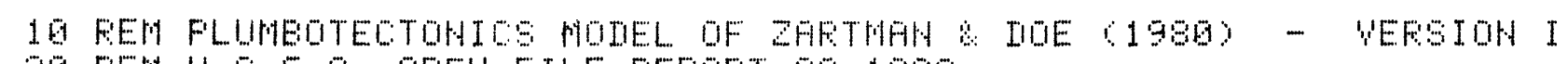

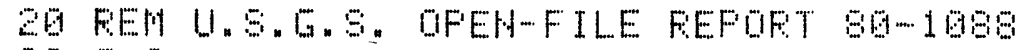

$30 \quad i=0$

$40 \quad I 1=0$

50 1 $12=0$

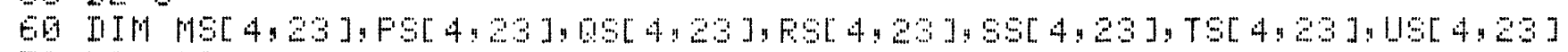

79 III AS[11] BS[11]:CS[11]

$80 L 1=0.155125$

$90 \mathrm{LE}=0.98485$

$190 \quad L 3=0.949475$

$110.90=10.36$

$120 \quad \%=12.12$

$13020=30.55$

$14 \mathrm{E} E 1=4$

$150 \mathrm{E} 2=1$

$16 \mathrm{E} E \mathrm{E}=1$

$17 \mathrm{~F} F 1=0.028$

$180 \mathrm{Fe}=0.754$

$190 \mathrm{Fg}=\mathrm{g} .21 \mathrm{~s}$

$200 \quad F 4=0.62$

$210 \quad F 5=0.788$

220 FE $=0.192$

$230 \quad F P=0.024$

$24 \mathrm{FB}=\mathrm{G} .854$

$25 \mathrm{Fg}=0.12 \mathrm{Z}$

$260 \quad \square=2.6$

$278 L=2, E$

$280 \quad T=4$

290 FIXEI 4

300 FOF I $=1$ TO 4

310 FOE $. I=1$ TO 23

320 … $[, 1]=0$

396 F $[1,1]=0$

340 Q $[I, 1]=0$

350 F $[1],]=0$

$360 \quad 5[I],]=0$

$370 \mathrm{~T}[\mathrm{I}, 1]=0$

390 ㄴ. $[, 1]=0$

390 HEXT I

469 HEYT I

410 M[ 1,1$]=800$

$426 \quad F[1,1]=38$

430 o[ 1,1$]=F[1,1] \div 80$

446 R $[1,1]=F[1,1] \div 0$

$458 \mathrm{~S}[1,1]=\mathrm{F}[1,1] \div 20$

$460 T[1,1]=1395$

470 u[ 1,1$]=349$

490 FOF: $I=1$ TO 11

490 FEFI $\mathrm{A}[\mathrm{I}]$

500 IATA 0.125,0.0625,0.03125,0.015025,0.0079125,0.0078125 
510 IATA 0.0078125,0.0078125,0.0979125,9.0078125,0.0078125

520 E[ I $]=0.3$

$53[$ [. I ] $]=0.1$

540 HEYT I

$550 \mathrm{k}=1$

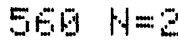

$579 \quad M=13$

$580 \quad 40=0$

590 11 $=0$

$606+12=0$

$610 \quad F Q=9$

$620 \mathrm{FI}=0$

$630 \quad P 2=8$

640 $09=0$

$650101=0$

$660 \quad 02=0$

E.9 $\mathrm{FO}=0$

$680 \times 1=0$

$690 \quad F 2=0$

$70080=0$

$71051=0$

$72052=0$

$730 \mathrm{~TB}=\mathrm{B}$

$740 \quad T 1=0$

$750 \mathrm{~T} 2=0$

$760 \quad 109=0$

$770 \quad 111=9$

$780 \quad 112=0$

$790 \mathrm{~m}[2.1]=\cdots[1.1] \div A[\mathrm{H}]$

800 FOF $J=2$ TO H

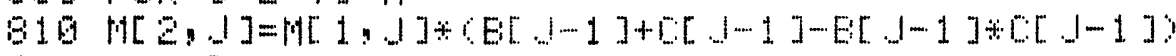

820 川1 $=4[2,1]+\cdots 1$

830 HEXT I

B4G FOR.$J 13$ TO M

B50 $M[2,1]=M[1,1]+[-1-12]$

$860+42=4[2,1]+12$

BTQ HEKT I

$88096=m[2,1]+m 1+42$

$890 M[3,1]=M b-C U+L)$

$900 M[4,1]=N[1,1]-M[2,1]+N[3,1]$

910 FOF $J=2$ TO

$920 \times[4,1]=\|[1,1]-\cdots[2,1]$

930 HEXT I

94 FOF $J=13$ TOM

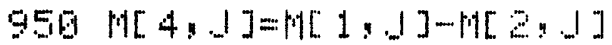

960 HEXT I

$970+1[4,1]=4$

$980 M[4, M]=L$

990 IF $G=3$ THEH 3299

1000 IF $G=4$ THEH 3460

$1610 \mathrm{~F}[2,1]=\mathrm{F}[1,1] \div \mathrm{A}[\mathrm{K}] * \mathrm{E} 1$

1926 FOR $J=2$ TO $H$

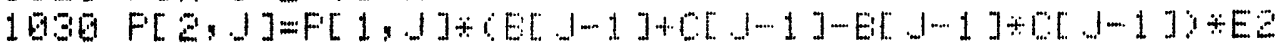

$1040 \mathrm{~F} 1=\mathrm{F}[2,1]+\mathrm{F} 1$

$1050 F[4,1]=F[1,1]-F[2,1]$

1960 HEXT J 


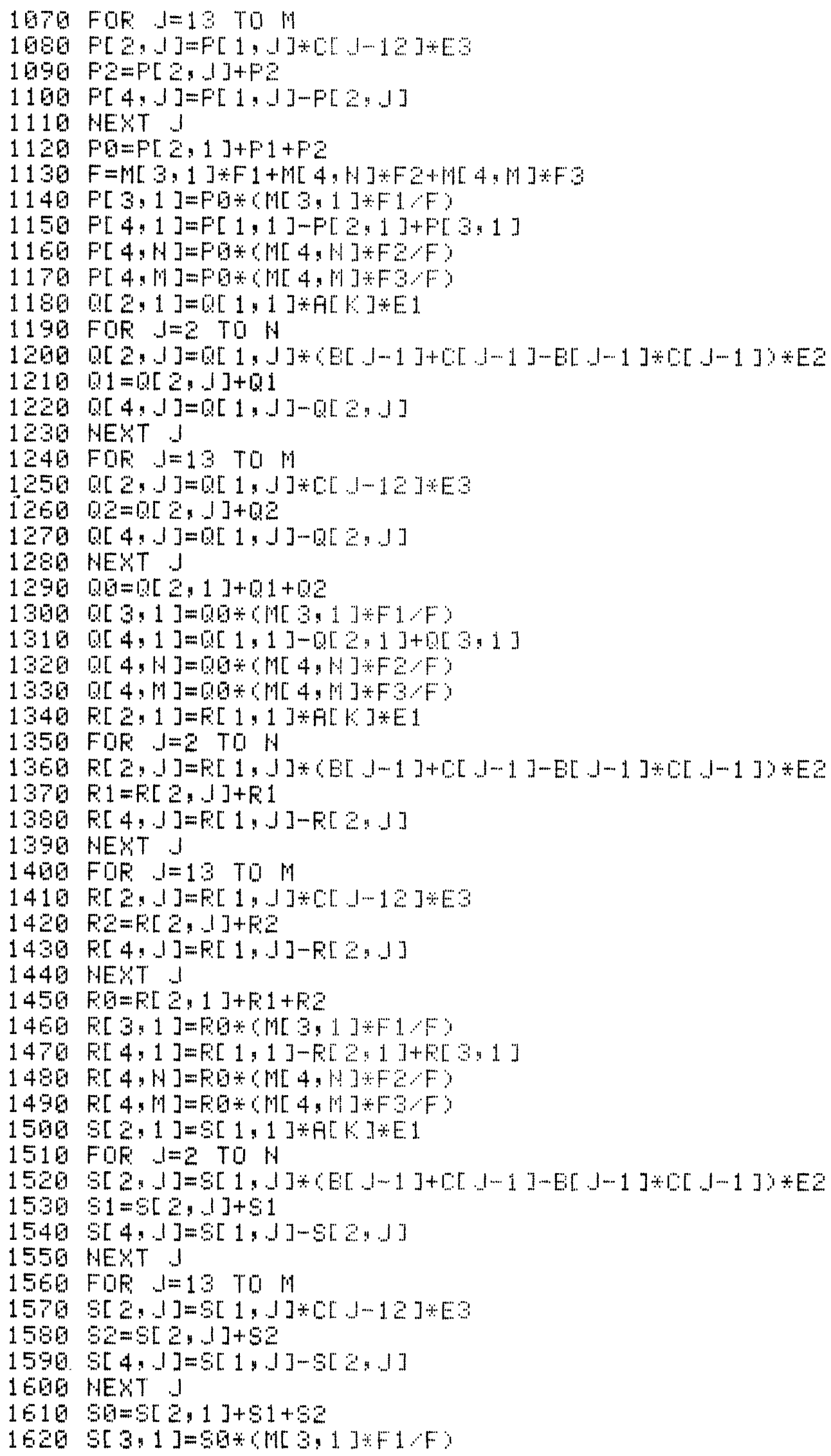


$16305[4,1]=5[1,1]-5[2,1]+5[3: 1]$

$16405[4, \mathrm{H}]=5 \mathrm{G} \div 6 \mathrm{H}[4, \mathrm{H}] \div \mathrm{F}, \mathrm{F}$

$16505[4, W]=56 \div W[4, W 7 \mathrm{~F} F \mathrm{~F}$

$16 E \mathrm{~T} T[2,1]=\mathrm{T}[1,1] \div \mathrm{H}[\mathrm{K}] \div \mathrm{E} 1$

$167 \mathrm{FQR}, \quad 1=2 \mathrm{TOH}$

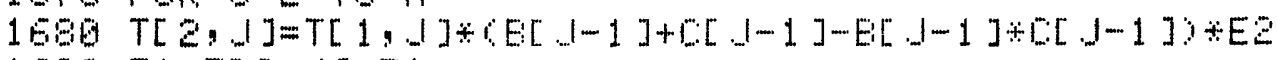

$1690 \quad T 1=T[2,1]+T 1$

$1706 \mathrm{~T}[4,1]=T[1,1]-T[2,1]$

1710 HEXT I

1726 FOF $.1=13$ TO

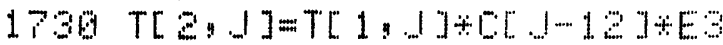

$1740 T 2=T[2,1]+T 2$

$1756 \mathrm{~T}[4,1]=T[1,1]-T[2,1]$

$17 E$ BE NET I

$1770 \quad T Q=T[2,1]+T 1+T 2$

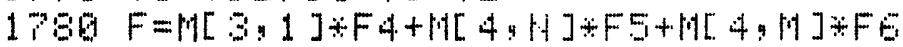

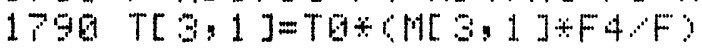

$1800 T[4,1]=T[1,1]-T[2,1]+T[3,1]$

$1610 \mathrm{~T}[4, \mathrm{H}]=\mathrm{TQ} \div 6 \mathrm{H}[4, \mathrm{H}] \mathrm{FS}, \mathrm{F}$

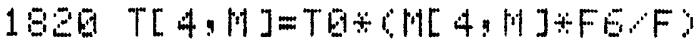

1830 U[ 2,1$]=U[1,1] \div \mathrm{F}[\mathrm{K}] \div \mathrm{E} 1$

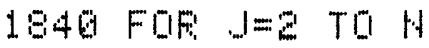

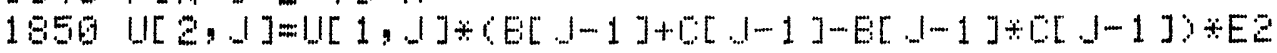

$1860 \quad 11=1[2,]+$.

$1870 \cup[4,1]=1 .[1,1]-U[2,1]$

1889 HEST J

1890 FOF.$=13$ TO

1960 U 2,$]]=U[1,1] \div[[]-12.] \div E S$

$1910 \quad 12=U[2, .1]+12$

$1920 \cup[4,1]=U[1, J]-U[2,1]$

1930 HEYT I

$1940 \quad 10=11[2,1]+111+112$

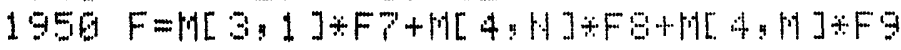

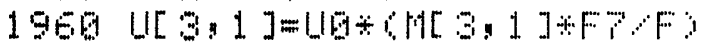

1970 U[ $4: 1]=0[1,1]-1][2: 1]+\cup[3: 1]$

1980 U $[4, \mathrm{H}]=110 \div 6 \mathrm{H}[4, \mathrm{H}] \div \mathrm{FG} \mathrm{F}$ )

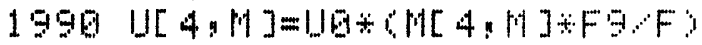

2000 IF $G=1$ THEH 2240

2010 IF $G=2$ THEH 2540

2020 IF $\square=5$ THEH $385 \mathrm{Q}$

209G IISF "DUTFUT DFTIOHO EHTEF 9 FOF LIST"

204 IHFIT $\square$

2050 IF $9 \# 9$ THEH 2150

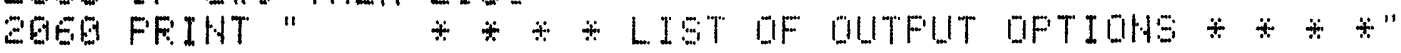

2079 FRIHT

2GEG FFIHT " 1 FEEFUOIF MASE AHII ISUTOFE COHTEHT"

2090 FFIHT " 2 ISOTOFIL: FHTIOS"

21G6 FFIHT " 3 T.MFH"

2110 FFIHT " 4 TMMIH"

2120 FFIHT " 5 FESEFUIIF OOHEHTFATIDHS"

2136 FFIHT

2140 G0TO 2030

2150 IF $G=1$ THEH 2210

2160 IF $G=2$ THEH $2 E 10$

2179 IF $5=3$ THEH 327

2180 IF $\square=4$ THEW 3440 
2199 IF $\square=5$ THEH 3799

2QUG FEM SUE-FOUTIHE FOF FESEFUUIF HASS AHD ISOTOFE COHTEHT

2210 FFIHT

220 FFIHT

2230 FEIHT

2240 FFIHT

2250 FFIHT

2200 FFIHT

2Z7G FFIHT

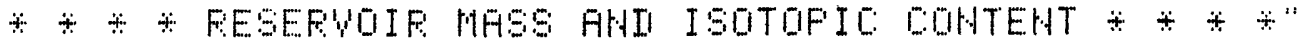

2280 FDE $J=1$ TO 23

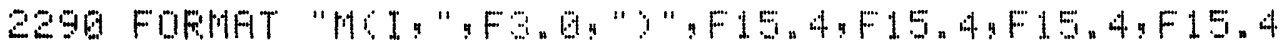

2300 HFITE $615,22903,1,1[1,1], 1[2,1], 14[3,1], M[4,1]$

2310 FOFHAT "FII:"F3.6."y",F15.4,F15.4,F15.4,F15.4

2320 UFITE $615,2310,1, F[1,1]$, F[2, 1$], F[3,1], F[4,1]$

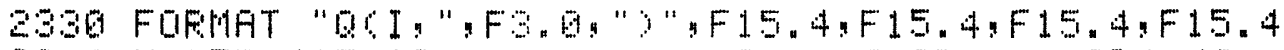

2340 HFITE $15,2350,1,0[1,1], 0[2,1], 0[3,1], 0[4,1]$

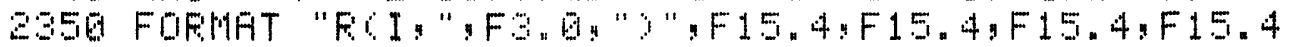

2360 WFITE $15,2350,1, F[1,1], F[2,1], F[3,1], F[4,1]$

237 FUFMAT "SCI,"FS. 1." "F15.4,F15.4,F15.4.F15.4

2380 WFITE (15,230)],5[1,1],5[2,1],5[3,1],5[4,1]

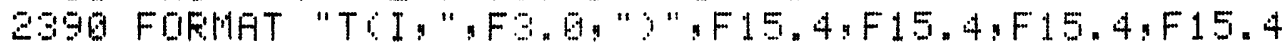

2400 HFITE $(15,2996), T[1,1], T[2,1], T[3,1], T[4,1]$

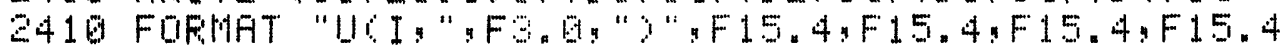

2420 WFITE $15,24163,1,1,1], 10[2,1], 1[5,1], 1[4,1]$

2436 FFIHAT

2440 NEST I

2450 FUF $J=1$ TO 23

2460 H $[1,1]=N[4,1]$

$2470[1,1]=F[4,1]$

$2480 T[1,1]=T[4,1]$

$2496 \cup[1,1]=1][4,$.

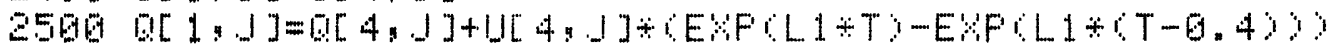

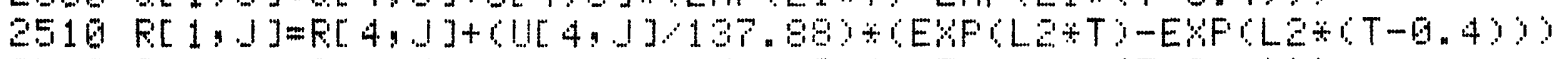

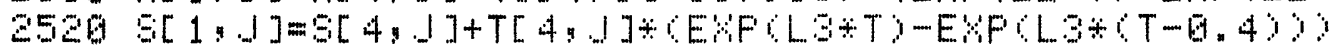

2536 HEYT I

$2540 T=T-0.4$

$2550 \quad r=k+1$

$2500 \quad H=N+1$

$2570 \quad M=M+1$

2580 IF $H=13$ THEH 4846

$2590 \quad 50 T 0 \quad 580$

2EG0 FEM SUE-FULTIHE FUF ISUTOFIC FHTIOS

2610 PRIHT"

2620 FPIHT

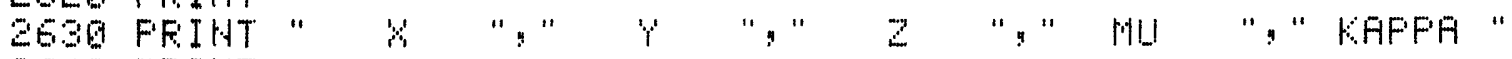

2640 FRIHT

EESE FRIHT "T="T"

2EEO FFIHT

EETG FFIHT "NHATLE"

2680 IF $F[1.1]=0$ THEH $272 \mathrm{E}$

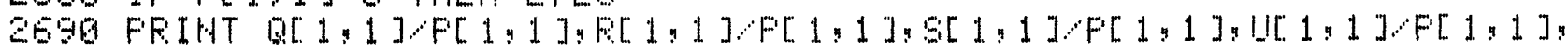

27GE FEIHT T[1:1] U[1:1]

2710 GOTO 2730

270 FFIHT $P[1,1], F[1,1], F[1,1], F[1,1], F[1,1]$

2730 FEIHT

270 FFIHT "LFFEF CFUST" 
2750 FUR $1=2$ TO 12

2700 IF $F[1,1]=0$ THEH 2809

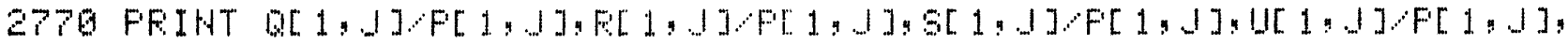

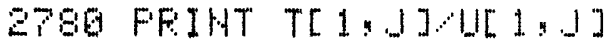

27901000 2810

2800 FRITT $F[1,1], F[1,1], F[1,1], F[1,1], F[1,1]$

2810 HEXT I

2820 FFINT

2330 FFIHT "LOHEF DFUST"

2840 FOF $\quad J=13$ TO 23

2950 IF $F[1,1]=0$ THEH 289

28E0 FFIHT $0[1,1]$ F[1, 1$], F[1,1] F[1,1], 5[1,1], F[1,1], 1][1,1], F[1,1]$,

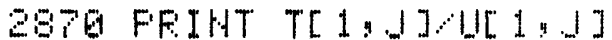

2800 G0T0 2900

2890 FFIHT $F[1,1], F[1,1]: F[1,1], F[1,1], F[1,1]$

2960 NEXT I

2910 FFIHT

2920 FFIHT "OFOLEHE"

2930 IF PG=6 THEH 2906

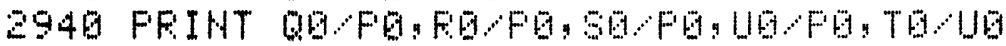

$2950 \quad 50 T 02970$

2950 PFIHT PG,FG, FG, FG,FG

2970 FRIHT

298G FRIHT "UFFEF EFUET COHTFE TUTEII TO QFOEEHE"

2990 IF F1=0 THEN 3020

3006 FEIHT 01,F1,F1,F1, :1,F1,U1,F1,T1.U1

3010 G0TO 3030

3020 FFIHT FI,F1,F1,F1,FI

3630 FRIHT

3040 FRIHT "LOWEF ERLET DOHAFIEUTEI TO OFOGEHE"

3050 IF $F 2=0$ THEH 3090

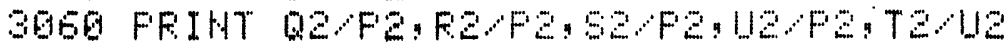

3670 FFIHT

3080 G0T0 3109

3096 FFIHT FE,FE.PZ,FE:FZ

3100 PFIHT

3110 FOR.$=1$ TO 23

3120 W[ 1,1$]=1[4,1]$

$3130 \mathrm{~F}[1 ; 1]=\mathrm{F}[4,1]$

$3140 T[1,1]=T[4,1]$

3150 U[ $[1 ; .1]=U[4 ; .1]$

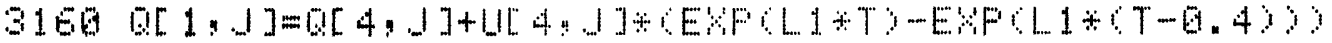

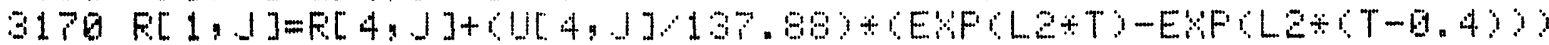

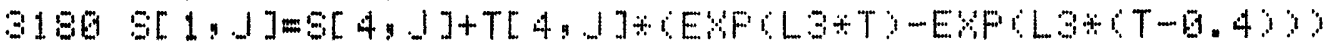

3190 HEQT I

$3200 \quad T=T-6.4$

$3210 \quad r=k+1$

$3220 \quad+1+n+1$

$3230 \quad M=川+1$

3240 IF $H=13$ THEH 4946

3250 190TO 500

3260 FEM SUE-FOUT IHE FOF T. WHO

3270 FFIHT

3280 FRIHT

3290 II $1=6[1: 1]-N[4: 1] 14 T+I 1$

$3306 \quad I 2=1[1 ; 1]-H[4,1]+[12$ 
$10 \mathrm{II}=\mathrm{II} 1 \mathrm{IL}$

3320 IF HC12 THEH 3340

3330 FFIHT "FUEFAGE FEE OF TOTAL CEUST: T:MAY = "II

3340 FOF.$=1$ TO 23

3350 M[1:.1]=m[4,1]

3360 HEXT I

$3370 T=T-6.4$

$3380 \quad r=K+1$

$390 \quad n=N+1$

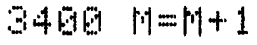

3410 IF $H=13$ THEH 4046

3420 GOTO 586

3430 FEM SUE-FOUTIHE FOF Ty WIH

3440 FFIHT

3450 FFIIVT

$3460 \mathrm{FQF} J=1$ TO 23

$3470 \quad \cdots[1, .1]=1[4, .1]$

3486 NEXYT I

$3490 \quad T=T-0.4$

$35010 k+1$

$3510 \quad \forall=k+1$

3520 .

3530 IF $H=13$ THEH 355

3540 50T0 580

$3550 \quad I=2$

$3560 \quad J=13$

$3570 \mathrm{A1}=\mathrm{B}$

$3580 \mathrm{AZ}=0$

$3590 \quad \mathrm{EI}=0$

$3600 \quad E 2=0$

3610 FDR $T=4$ TO 9 STEF -0.4

$3620 \quad A 1=14[4, I] \div T+A 1$

$3630 \quad A 2=M[4,1] \div T+F 2$

$3640 \quad E 1=N[4, I]+E 1$

$3650 \quad E 2=n[4,1]+E 2$

$3600 \quad I=I+1$

$3670.1=1+1$

$3 E 90$ HEXT T

$3690 \quad A=6 A 1+H 23 \quad 61+E 2$

$3760 \mathrm{E}=\mathrm{F} 1 \mathrm{EI}$

$3710 \mathrm{D}=A 2 \mathrm{EZ}$

37EG FEIHT "AWEFALE FEE OF TOTHL OFUST: T.MIHCT.C.

3730 FFIIHT

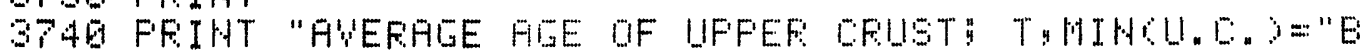

3750 FFIHT

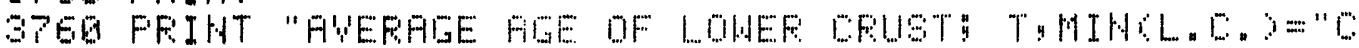

379 IF $N=13$ THEH 4040

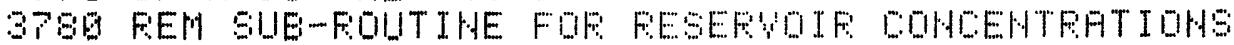

3796 FFILIT

3960 FFIHT

3910 FFIHT

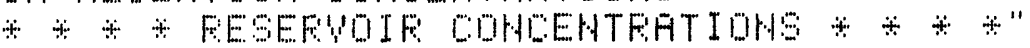

3820 FFIHT

3830 FFIHT

3840 FFIHT

3650 FDF.$=1$ TO 23

$3860 M[1,1]=M[4,1]$ 


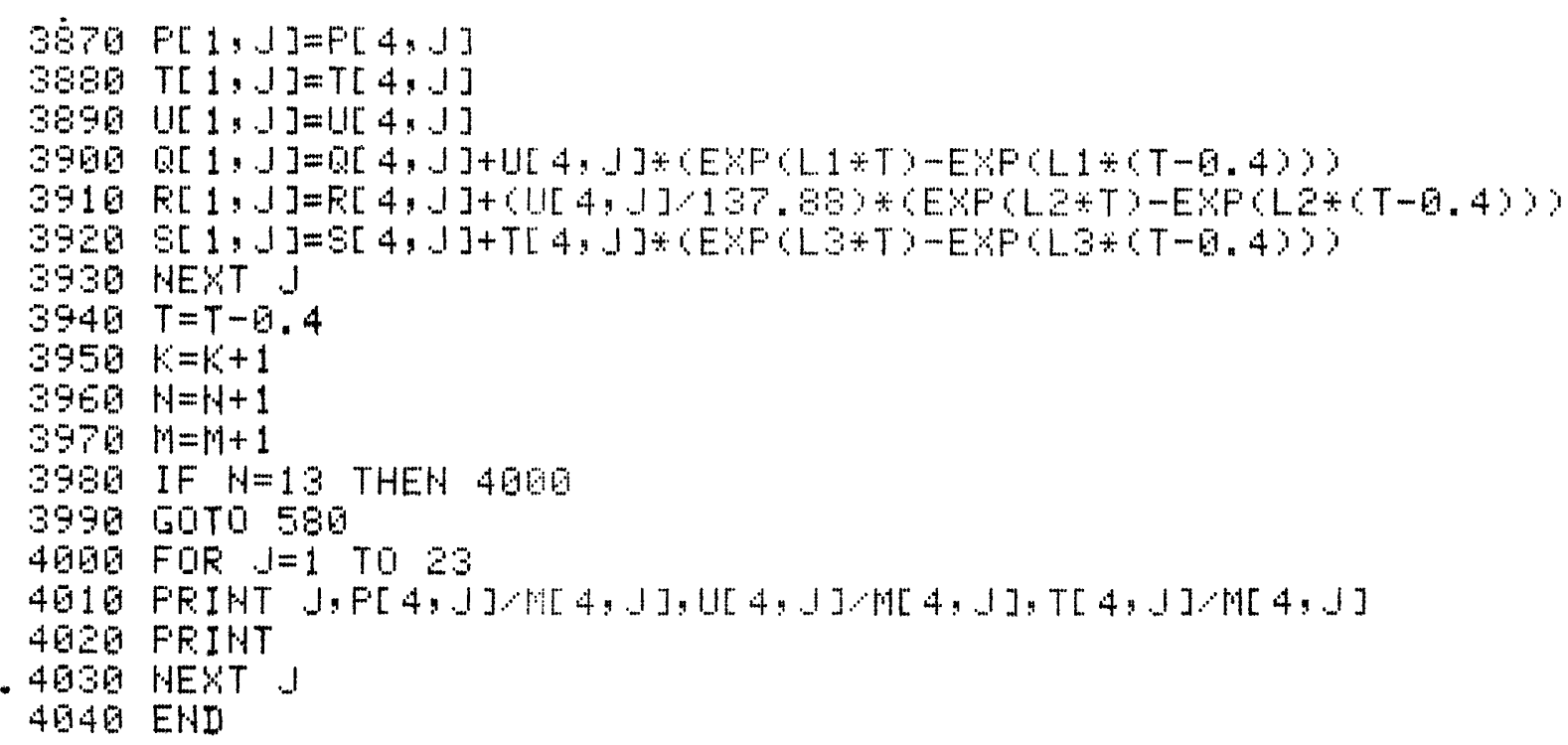




\section{AFFEHIIY A: FLUNEOTECTOHIOS NOLEL CVERSIOH I? \\ ᄅ. LISTIHE OF SUEROUTIHE FOF HEHH ALE OF ISOTOFES IH CEUST}

19 FEN FLUWEOTEOTOHIOS WOIEL OF ZAFTHAH \& DOE 1980 - WEFSIOH I

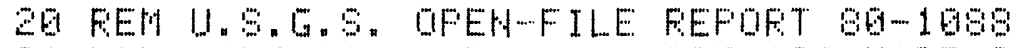

30 II M M[4,23],FS[7,23],TS[7,23],US[7,23]

40 III FS[11],ES[11], ES[11], ISE 3411$]$

50 FOF $I=1$ TO 11

EQ REEI A[I I

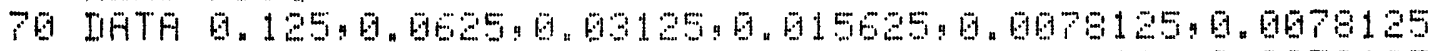

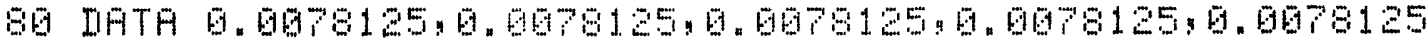

$90 \mathrm{E}[\mathrm{I}]=0.3$

100 [L[I] $]=0.1$

110 HEST I

$120 \quad E 1=4$

$130 \quad E Z=1$

$149 \quad E 3=1$

$150 \quad F 1=0.028$

$160 \quad \mathrm{FE}=0.754$

$170 F 3=0.218$

$150 \quad F 4=0.02$

$190 \quad F 5=0.788$

$200 \mathrm{FE}=0.192$

$210 F 7=0.024$

220 FB=0.854

$230 \quad F 9=0.122$

$240 \quad 1=2.6$

$250 \mathrm{~L}=2.6$

2EG FISEI 4

276 FOF $Z=2$ TU 12

280 FOF I=1 TO 4

290 FOF $J=1$ TO 23

306 W $[1,1]=6$

310 HEYT I

320 HEXT I

330 FOE I $=1$ TO $?$

340 FOF $y=1$ TO 23

350 F $[I, 1]=6$

260 T $[1,1]=0$

$37011,1]=0$

380 HEXT I

390 HEXT I

406 Hin $[1,1]=800$

$410 F[1,1]=30$

420 T[ 1,1$]=1335$

430 U[ 1,1$]=349$

$440 \mathrm{k}=1$

$450 \quad H=2$

460 川 $=13$

$470 \mathrm{~T}=4$

480 1 $19=0$

$49011=0$

$50012=0$ 


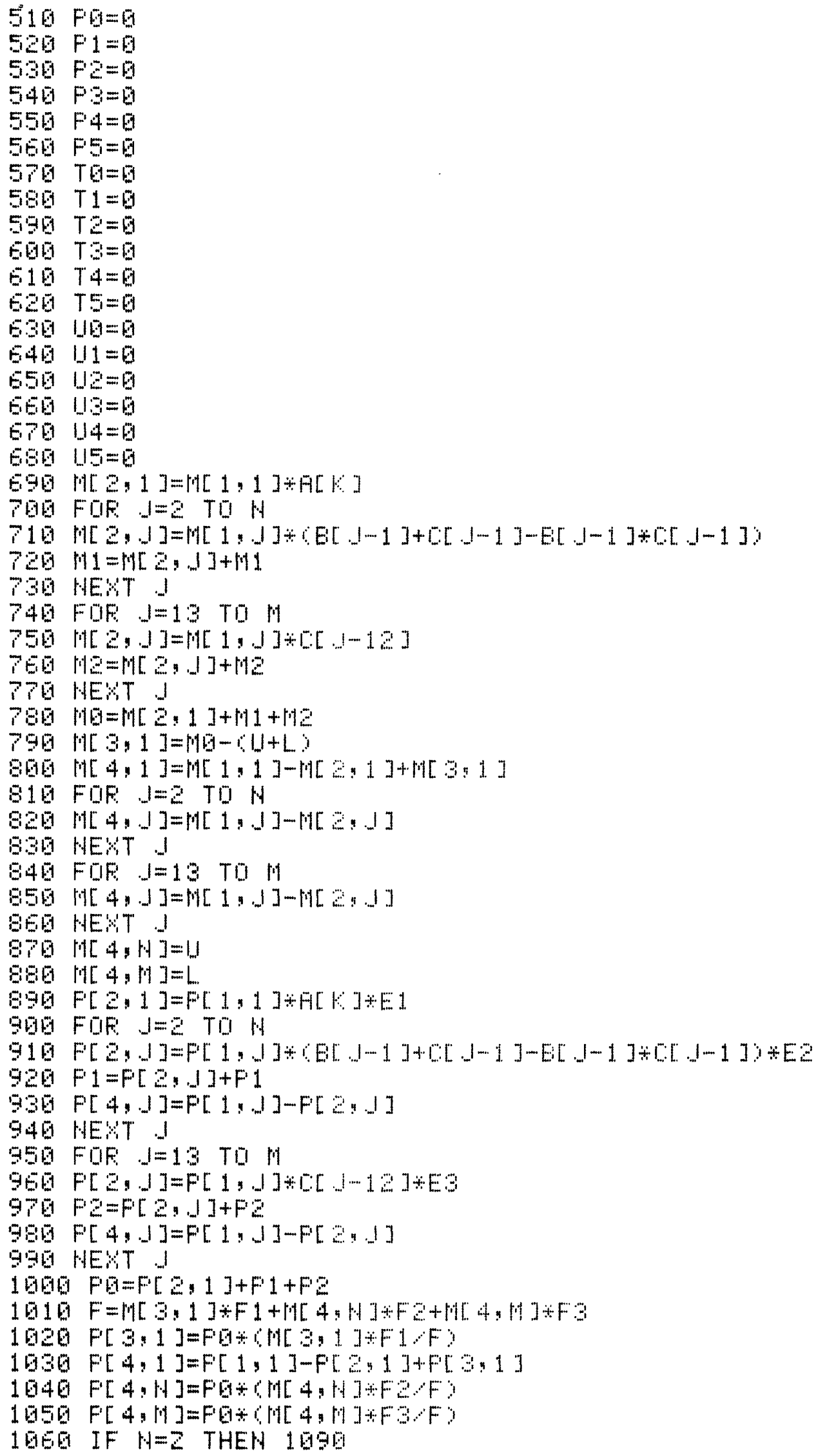


1070 IF HO THEH 1126

1980 G0TO 1250

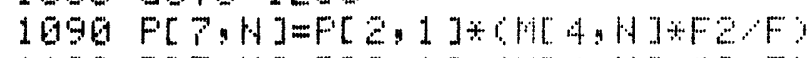

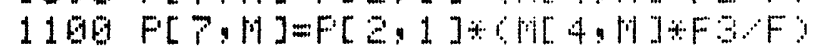

1110 GOTO 1250

$112 \mathrm{GOF} . J=2 \mathrm{TO} \mathrm{H}$

$1130 \mathrm{~F}[\mathrm{E}, 1]=F[5,1] \div(\mathrm{E}[1-1]+[1,1-1]-E[1-1] \div[.1-1]) * E 2$

$1140 \mathrm{~F} 4=F[E, .1]+F 4$

$1150 \mathrm{~F}[7,1]=F[5,1]-F[0,1]$

$11 E \mathrm{~B}$ HEWT I

$1170 \mathrm{FOF} . \mathrm{I}=13 \mathrm{TO}$ W

$1180 F[6,1]=F[5,1] \div[[1-12] \div E 3$

$1190 \quad F 5=F[6,1]+P 5$

$1200 F[7,1]=F[5,1]-F[0,1]$

1210 NEXT I

$1220 \quad F 3=F 4+F 5$

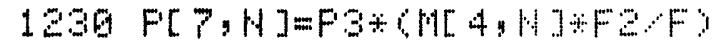

$1240 \mathrm{~F}[\mathrm{P}, \mathrm{H}]=\mathrm{F} 3 * 6 \mathrm{H}[4, \mathrm{M}]+\mathrm{F} 3 \mathrm{~F} \mathrm{C}$

$1250 T[2,1]=T[1,1] \div \mathrm{H}[\mathrm{F}] \div \mathrm{E} 1$

$12 E \mathrm{FOF} . \mathrm{I}=2 \mathrm{TO}$

$1270 \quad T[2],]=T[1],] \div(\mathrm{E}[1-1]+[-1-1]-\mathrm{E}[1-1] \div[.1-1]) \div E 2$

$1230 \quad T 1=T[2 ;, 1]+T 1$

$1290 T[4,1]=T[1,1]-T[2 ; 1]$

1300 HEWT I

$1310 \mathrm{FQF} . \mathrm{I}=13 \mathrm{TOH}$

$1320 \quad T[2, .1]=T[1, .1] \div[1.1-12] \div \mathrm{E}$

$1330 \quad T 2=T[2,1]+T 2$

$1340 T[4,1]=T[1,1]-T[2,1]$

1350 HEYT I

$1360 T 0=T[2,1]+T 1+T 2$

$1370 \mathrm{~F}=M[3,1] \div F 4+1[4,1] \div F 5+14[4,4] \div F E$

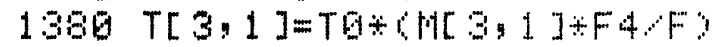

$1390 T[4,1]=T[1,1]-T[2,1]+T[3,1]$

$1400 \quad T[4,1]=T Q \div 6[4, H]+5 \mathrm{~F}$

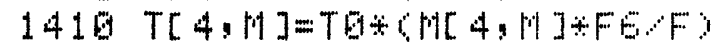

1420 IF $H=2$ THEN 1450

1430 IF $H Y Z$ THEH 1480

144060701610

1450 T[ 7,4$]=T[2,1] * 6,[4, H] \div F, F)$

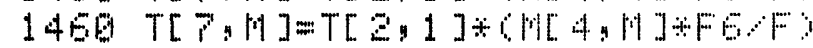

1470 GOTO 1610

$1480 \mathrm{FOR}, \mathrm{I}=\mathrm{2}$ TO $\mathrm{H}$

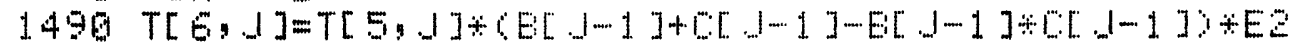

$15000 T 4=T[E, J]+T 4$

$1510 T[7,1]=T[5,1]-T[E, 1]$

1520 HEXT J

1530 FOR $J=13$ TO $M$

$1540 T[6,1]=T[5,1] *[1,1-12] * E 3$

$1550 \quad T 5=T[E, 1]+T 5$

$1560 \quad T[P, J]=T[5,1]-T[6,1]$

1570 HEXT J

$1580 \mathrm{~T} 3=\mathrm{T} 4+\mathrm{T} 5$

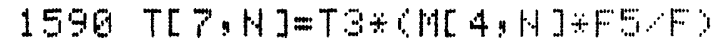

$1600 \mathrm{~T}[\mathrm{P}, \mathrm{M}]=\mathrm{T} 3 *(\mathrm{M}[4, W] * \mathrm{FE} \mathrm{F}$

1610 U[ 2,1$]=\|[1,1] * \mathrm{~A}[\mathrm{~K}] * \mathrm{E} 1$

1620 FOR $. J=2$ TO H 


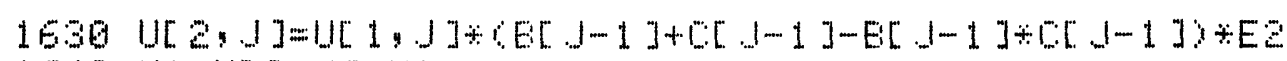

$1640 \quad 11=1[2,1]+11$

1650 U[ 4,1$]=U[1,1]-U[2,1]$

$16 E 0$ NEYT J

$167 \mathrm{FOF} . J=13 \mathrm{TD} M$

1580 U[ 2,1$]=U[1,1] \div[[1-12] * E$

$1690 \quad \cup 2=U[2 ; 1]+42$

1706 U. 4,1$]=U[1,1]-U[2,1]$

1710 HEXT I

1726 U6= $152: 1]+11+42$

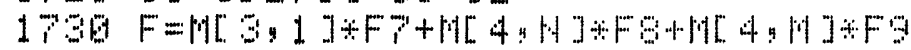

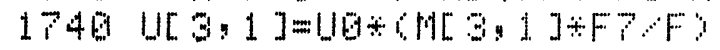

1750 U[ $4: 1]=0[1 ; 1]-U[2 ; 1]+1[3 ; 1]$

1780 U[ $4: 4]=60 \div 64[4,4] \div \mathrm{FE} F$

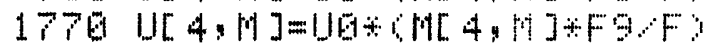

1780 IF $H=Z$ THEN 1810

1790 IF HD THEH 1848

1800 G070 1950

1810 U[ 7,4$]=U[2,1] \div 6 \mathrm{HL}, 4] \div \mathrm{FBF}$

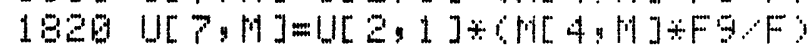

$183050 T 01980$

$1840 \mathrm{FOF} J=2$ TO H

1850 U[ $E, 1]=U[5, J] \div 6[1-1]+[.1-1]-E[1-1] \div[1-1]) \div E Z$

$1806 \quad 114=10[6,1]+114$

$1870 \cup[7 ; .1]=1[5 ; .1]-\cup[6,1]$

1880 HEKT 1

$1890 \mathrm{FOF} .=13 \mathrm{TO}$ W

1960 U[ 6,1$]=[15,1] \div[.1-12] \div E S$

$1910 \quad 15=4[6,1]+45$

1920 U $7,.]=U[5, J]-4[6,1]$

$1930 \mathrm{NEXT} J$

$1940 \quad 03=44+45$

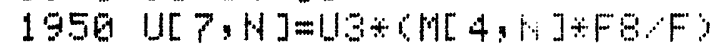

1960 U[ $7, M]=03 *(M[4, M] * F 9 . F)$

1970 FOF $\quad J=1$ TO 23

1980 FOR $J=1$ TO 23

1990 M[ $1, .1]=m[4,1]$

$2600 \mathrm{~F}[1, J]=F[4,1]$

$2010 F[5,1]=F[7,1]$

$2020 \quad T[1,1]=T[4, J]$

$2030 \quad T[5, J]=T[7,1]$

$2040 \quad U[1, J]=U[4, J]$

$2045 \quad u[5,1]=U[7,1]$

2050 HEXT I

$2060 \quad T=T-0.4$

$2070 \quad k=k+1$

$2080 \quad H=N+1$

$2096 \quad M=N+1$

2100 IF $H=13$ THEH 2120

2110 G0TO 480

$2120 \quad \mathrm{~A}=0$

$2130 \mathrm{E}=0$

2140 FDR $\quad J=2$ TO 12

$2150 \quad A=P[7 ; 1]+A$

$2160 \quad E=P[7, j+11]+E$

2170 HEXT J

2180 II $[, Z-1]=\mathrm{A}+\mathrm{B}$ 
2190 FOF $I=Z$ TO 12

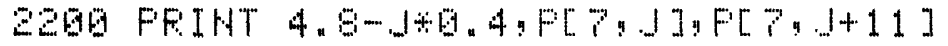

2210 NEXT I

2220 FFI I HT

236 FFIIT

2246 FRIHT

250 FFIHT

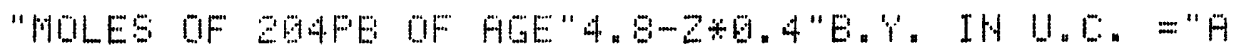

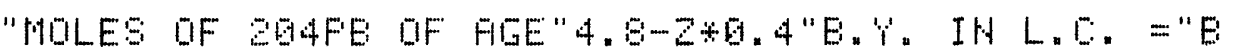

$2 E B$ FFIHT

$2280 \quad \mathrm{H}=0$

$2290 \quad E=0$

$2300 \mathrm{FOF} . J=2$ TO 12

$2310 \mathrm{H}=\mathrm{T}[\mathrm{P}, \mathrm{M}]+\mathrm{H}$

$2320 \mathrm{E}=\mathrm{T}[\mathrm{P}]+11],+\mathrm{E}$

2330 NEXT I

2346 II $[Z, Z-1]=F+B$

2350 FOF $I=2$ TD 12

2360 FFIHT 4.5-JiG.4:T[7. I]:T[7.1+11]

2370 NEXT I

2380 FRIHT

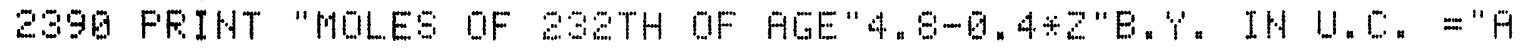
2406 FFIHT

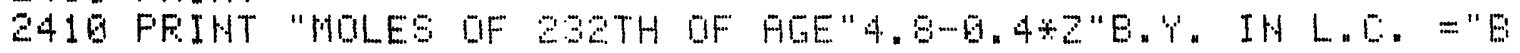
2420 PFINT

2430 FRIHT

$2440 \quad H=0$

$2450 \mathrm{E}=0$

2460 FOR $J=2$ TO 12

$2470 \quad A=U[7, J]+A$

$2480 E=U[7,1]+11]+B$

2490 HEXT I

2500 II $[3,2-1]=\mathrm{H}+\mathrm{E}$

2510 FOR $J=Z$ TO 12

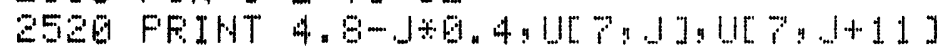

$2530 \mathrm{HEXT}$.।

2540 FFIHT

2550 FFIHT "MULES OF 25 U OF ALE"4.9-0.4*2"E.\% IH U.C. ="H

2560 FFIHT

2570 FRIHT "MOLES OF 23 BU QF AGE"4.8-0.4*2"E.\%. IH L.C. = "E

2580 FFIHT

2590 FRINT

2600 HEXT 2

2610 FFIHT

262G PFINT

2630 PRIHT

$2640 \quad 01=0$

2650 II $1=0$

$2600 \quad 02=0$

2670 II $=0$

$2680 \quad 03=0$

$2690 \quad 13=0$

2700 FOF $J=1$ TO 11

$2710 \quad[1=I[1,1] \div(4.4-0.4 * 1)+[1$

2720 II $=I[1,1]+I 1$

$2730 \quad[2=\pi[2,1] * 4.4-0.4 * 1)+[2$

$2740 \quad I 2=I[2,1]+D 2$ 
$2750 \quad[3=\operatorname{II}[3.1] \div 4.4-6.4 \div .13+[3$

$2760 \quad$ II: $=[1[3: .1]+[13$

$27 \%$ HEWT I

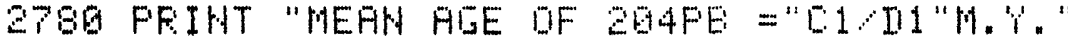

2796 FFIITT

2800 FRINT "MEAH RLE OF $232 T H=" Q Z Z \mathrm{IZ}$ "M. $\%$."

2810 FRIHT

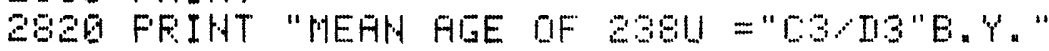

2830 FRINT

2840 FRINT

2850 FFIHT

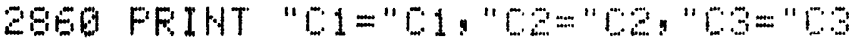

2870 FRIHT "II="II, "IE="IL, "IS="IS

$2880 \mathrm{EHI}$ 
Mass distribution for the various groupings of reservoirs as a function of t1me. $\frac{u(1)}{4.0}$ and $\frac{l(1)}{4.0}$ are also given to illustrate the decrease in size of crustal reservolrs with successive orogenies. [All masses in units of $10^{24} \mathrm{~g}$ ]

\begin{tabular}{lcccccc}
\hline 1 & $t\left(b_{.} y_{.}\right)$ & $M_{t}^{\frac{m}{t}}$ & $\sum M_{t}^{u(i)}$ & $\sum M_{t}^{l(1)}$ & $M_{4.0}^{u(1)}$ & $M_{4.0}^{l(1)}$ \\
\hline 0 & 4.0 & 800.0 & 0.000 & 0.000 & 0.000 & 0.000 \\
1 & 4.0 & 794.8 & 2.600 & 2.600 & 2.600 & 2.600 \\
2 & 3.6 & 790.8 & 4.238 & 4.940 & 1.638 & 2.340 \\
3 & 3.2 & 787.7 & 5.270 & 7.046 & 1.032 & 2.106 \\
4 & 2.8 & 785.1 & 5.920 & 8.941 & .650 & 1.895 \\
5 & 2.4 & 783.0 & 6.330 & 10.647 & .410 & 1.706 \\
6 & 2.0 & 781.2 & 6.588 & 12.183 & .258 & 1.535 \\
7 & 1.6 & 779.7 & 6.750 & 13.564 & .163 & 1.382 \\
8 & 1.2 & 778.3 & 6.853 & 14.808 & .102 & 1.244 \\
9 & 0.8 & 777.1 & 6.917 & 15.927 & .065 & 1.119 \\
10 & 0.4 & 776.1 & 6.958 & 16.934 & .041 & 1.007 \\
11 & 0.0 & 775.2 & 6.983 & 17.841 & .026 & .907 \\
\hline
\end{tabular}




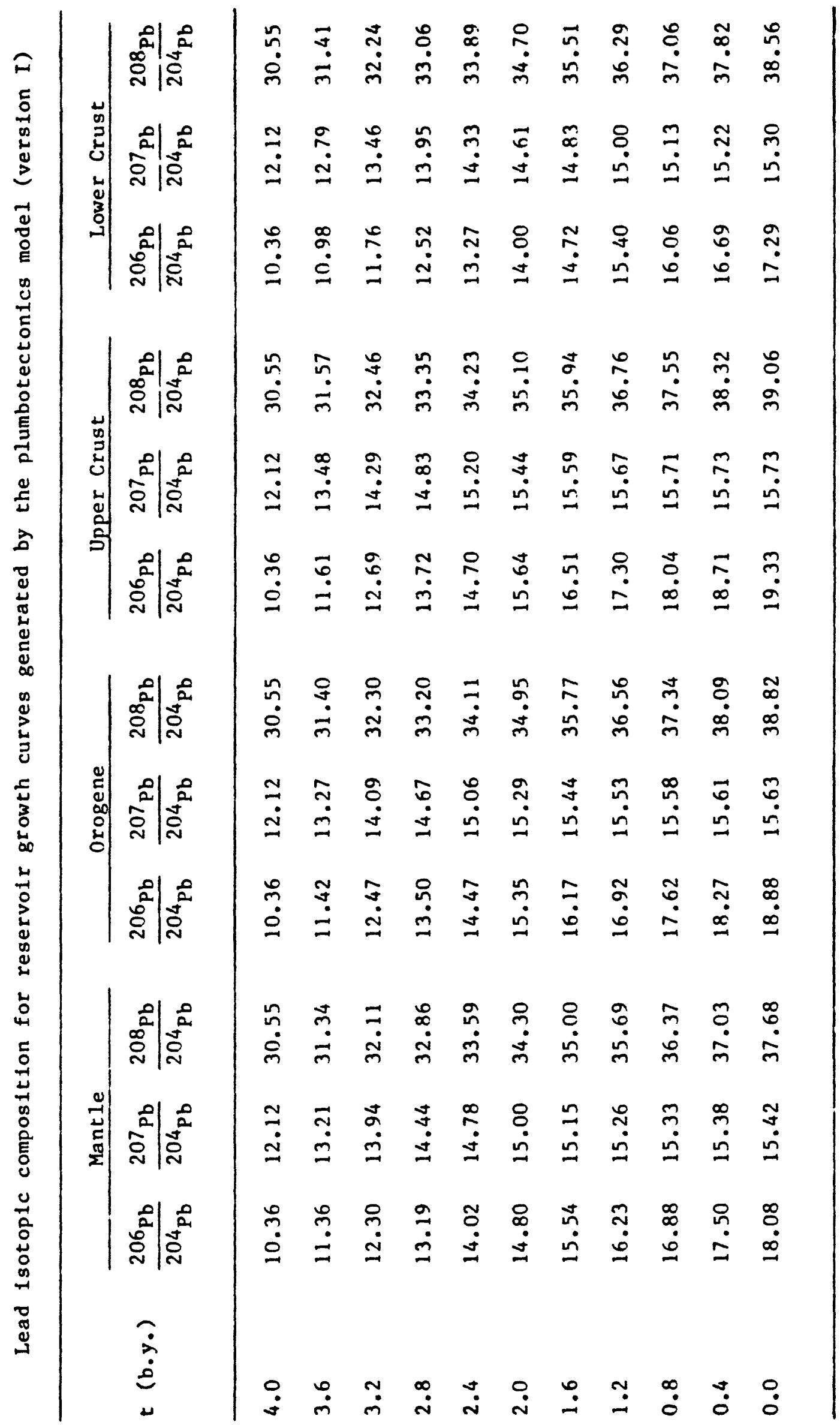




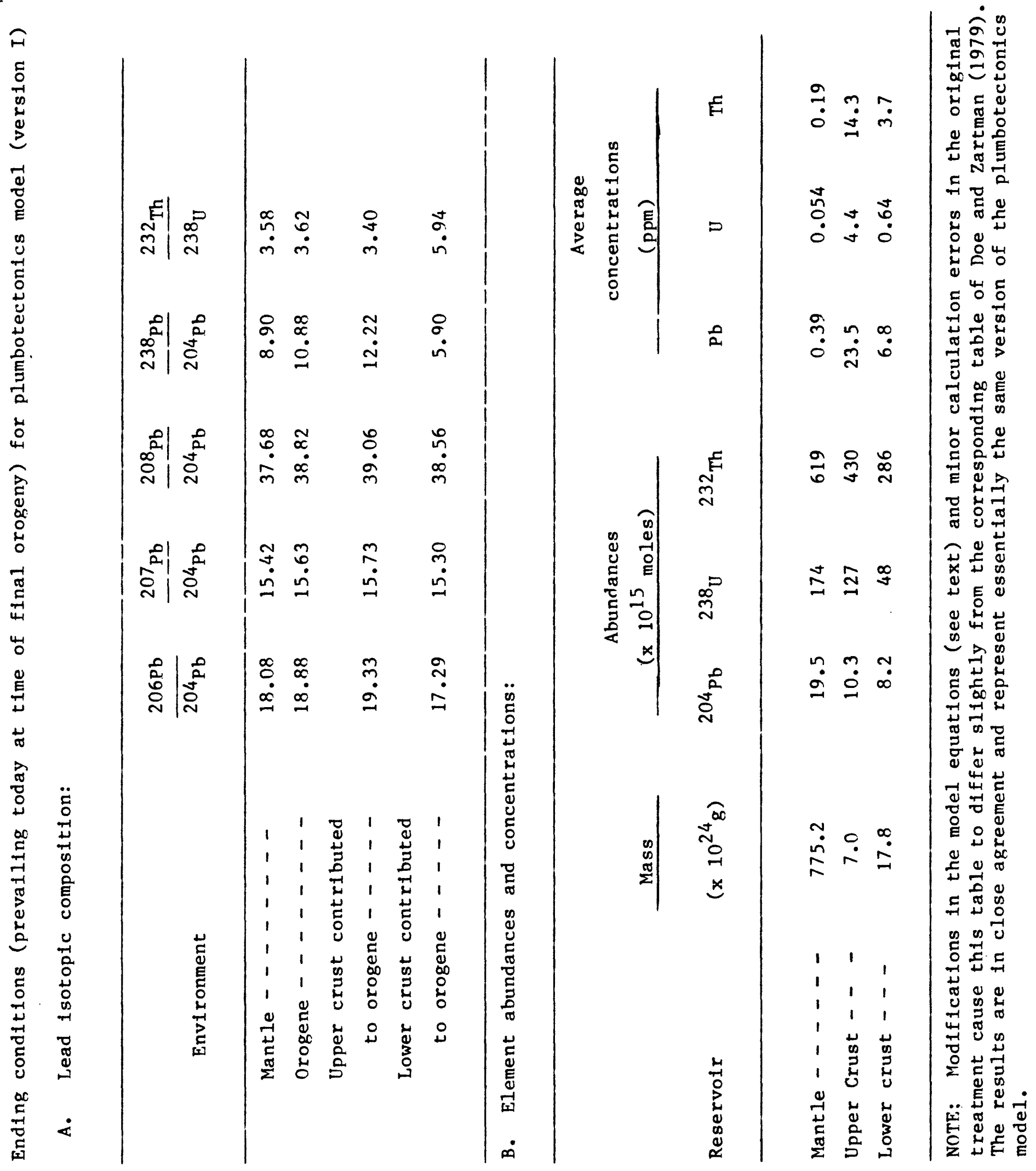


AFFEHII E: FLUHEOTELTOHISS MOIEL CUEFSIDH II

1. LISTIH OF MFIH FFOLFAN CFFOLFAH IS SINILAF TO THAT OF WEFSIOHA I GIVEH IH AFFEHIIY A EXEEFT THAT THE FQLLOHIHI LIHES SHOULI EE SUESTITUTEI FOF THE EQUIUALEHT LIHES OF UEFEI 매 I?

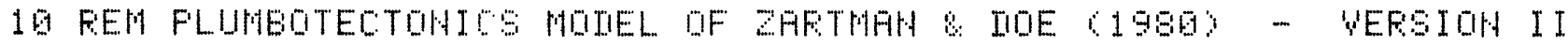

$110 \quad 3=10,32$

$13020=30.56$

$170 \mathrm{~F} 1=0.636$

$196 \mathrm{FZ}=\mathrm{G}, 7 \mathrm{ZZ}$

$190 \quad F 3=0.222$

$210 \quad F 5=6.766$

$220 \mathrm{FE}=\mathrm{G}, 194$

$2469=9.952$

$250 \quad F 9=6.124$

$426 F[1,1]=37$

$460 T[1: 1]=1316$

470 U[ 1,1$]=344$

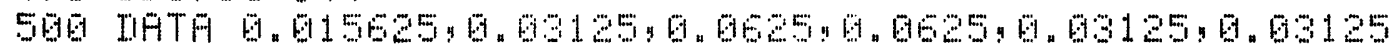

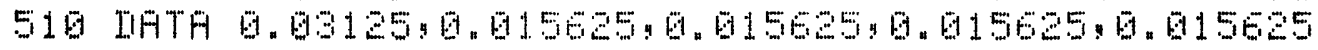

2. LISTIHG OF SUEEOUTINE FOF HEHA ALE DF ISOTOFES IH OFUST CFFOLFH IS STHILAF TO THFT OF VEFSIOH I GIUEN IH AFPEHII H F EDEFT THAT THE FOLLOHIHI LIHES SHLILI BE SUEST ITUTED

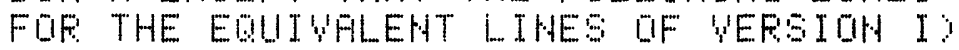

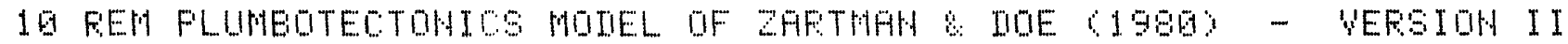

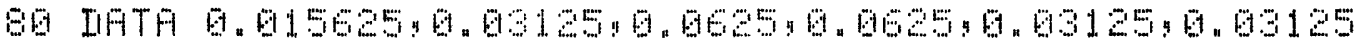

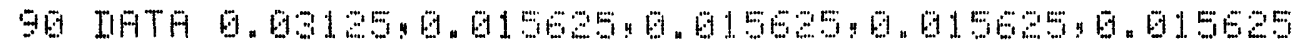

$160 \mathrm{~F} 1=0.0 \mathrm{6}$

$176 F=6.72$

$190 \quad F 3=9,22$

$200 \quad F 5=6.786$

$210 \mathrm{FE}=0.194$

$230 \mathrm{FB}=0.852$

$24 \mathrm{FG}=\mathrm{G}, 12 \mathrm{C}$

$420 \mathrm{~F}[1,1]=3$

$430 \mathrm{~T}[1,1]=1316$

440 니 1,1$]=344$ 


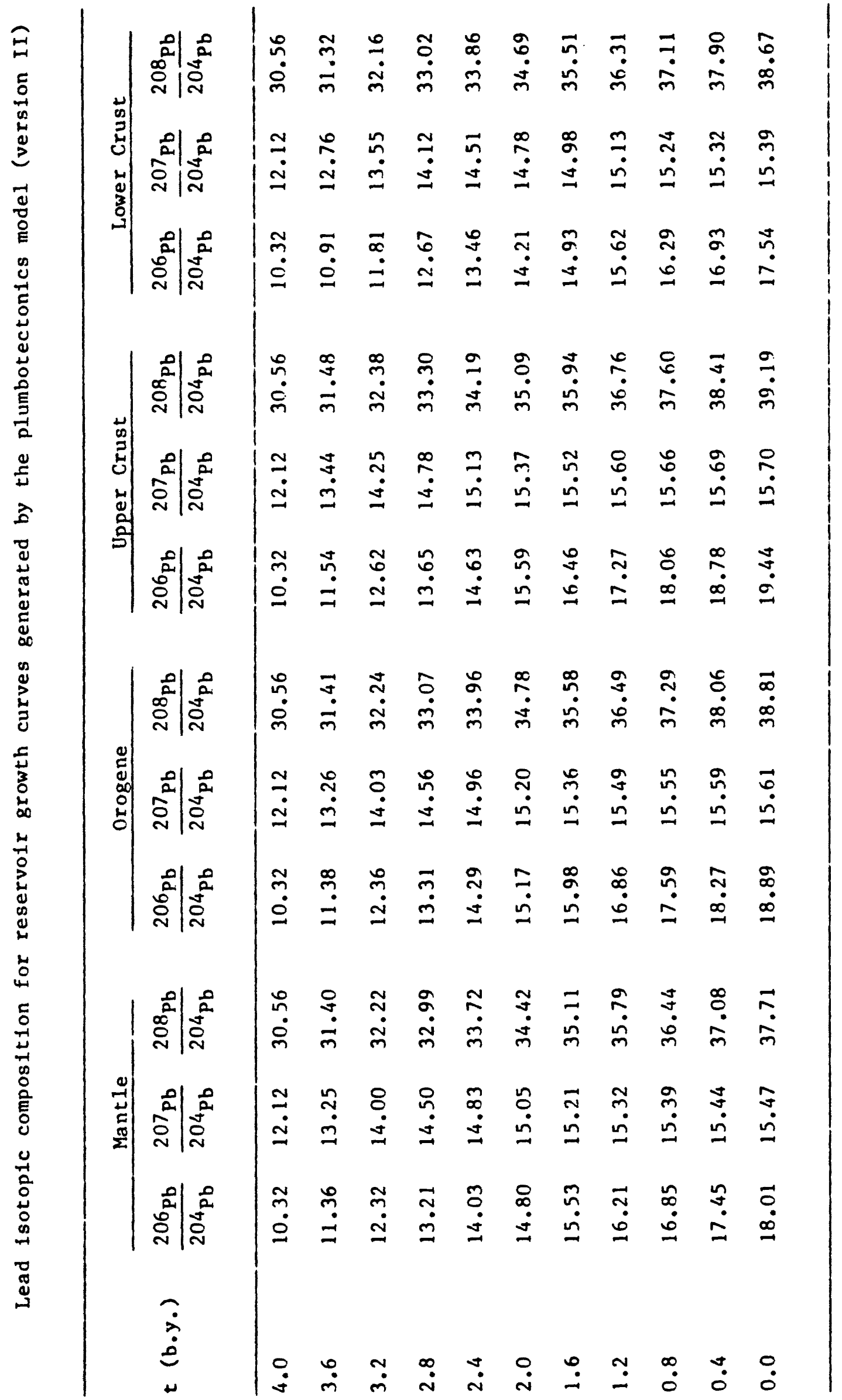

